\section{“ANTISTIN" IN DERMATOLOGY} BY

JAMES OVERTON, M.D., B.Sc., M.R.C.P. Honorary Chief Assistant, Skin Department, Leicester Royal Infirmary

Research by K. Miescher and co-workers on antihistamines led to the synthesis of " antistin," a substance which chemically has the formula $2 \mathrm{~N}$-phenyl-N-benzyl-aminomethylimidazoline.

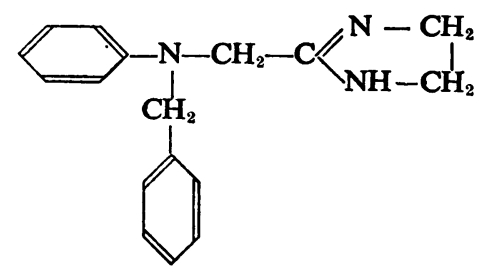

The antistin salts used are all white crystalline powders. The hydrochloride, which is $2 \%$ soluble in water, is used in the tablets. The methanesulphonate is readily soluble in water, and is given parenterally. Antistin sulphate has a solubility of about $4 \%$ in water, and is employed as a local application to the mucous membrane in hay-fever.

\section{Pharmacology}

The pharmacological properties of antistin have been investigated by Meier and Bucher $(1946 \mathrm{a}, 1946 \mathrm{~b})$ in the usual animal experiments employed to ascertain the efficacy of this type of drug. Anaphylactic symptoms were entirely prevented by previous medication with antistin in rabbits sensitized to egg albumin; antibody production appeared to be increased when elicited in conjunction with the administration of antistin; addition of antistin to the liquid in which isolated organs were immersed suppressed or prevented anaphylactic contraction (Schulz-Dale's experiment). The antagonism of antistin to histamine was shown in the isolated perfused posterior extremity of the rabbit and in the isolated intestine of the guinea-pig; the fatal consequences from histamine-induced bronchospasm in the guinea-pig were prevented by premedication with antistin. Its toxicity was found to be relatively low, and cumulative action slight. Brack (1946) demonstrated its peripheral action on the vasomotor system in man by the effects of local application in scratch-tests with histamine, diethylmorphine, and simple trauma ; he observed suppression of the irritation and a reduction in the size of weal formation. Antistin did not reduce the irritation which followed the application of adrenaline in this scratch-test technique. Staub (1946) found that the temporary increase in the histamine content of the blood in man after the slow intravenous administration of $0.2 \mathrm{mg}$. of adrenaline was diminished or abolished by the previous administration of antistin.

\section{Clinical Trials}

Schindler (1946) attempted a clinical evaluation of antistin in a mixed series of conditions associated with pruritus. Among four patients suffering from jaundice due to infective hepatitis, three were relieved in one to three days, and in one there was no conclusive result. In two patients with carcinoma of the duodenum relief was obtained after two and six days' medication respectively. One patient with icterus of unknown aetiology was free from pruritus after two days' treatment. Senile pruritus was relieved in one patient, but in another, after a temporary amelioration, the drug proved ineffective. An old man with chronic generalized pruritus experienced relief only while taking antistin tablets. Itching associated with lymphogranulomata was relieved in one patient, but in another, after an initial period of partial relief, the drug proved useless. Relief was obtained after three days' treatment in a patient suffering from plasmocytoma. In a patient with carcinoma of the pancreas immediate success was followed by subsequent failure. The result of treating one patient with drug-pruritus was unconvincing.

Nine cases of acute urticaria-three of which were due to drugs, two to sera, two to foodstuffs, and one following ultra-violet light therapy-were rapidly relieved by antistin. One patient suffering from recurrent uricaria obtained speedy relief from a relapse, whereas another patient who also suffered from myocardial degeneration experienced little benefit. It proved ineffective for two patients with erythema nodosum.

Schindler found that dosage was purely empirical; he administered up to $300 \mathrm{mg}$. daily intravenously, intramuscularly, or subcutaneously, and up to $600 \mathrm{mg}$. by mouth. He tried the effect of a 200-mg. dose intravenously in a normal control. The only ill effect suffered was a sense of warmth in the head, lasting about five minutes. Doses of $300 \mathrm{mg}$. daily for 10 days in four normal controls did not produce any unpleasant symptoms, nor were the pulse, blood pressure, or respiratory rate affected. Occasionally, when the subcutaneous route was used, a painful nodule persisted for a few days at the site of injection.

Brack (1946) has carried out more extensive trials on about a hundred patients suffering from various skin disorders. Nine patients with acute urticaria and four pat:ents with " cold" urticaria manifested rapid relief. Seven patients with chronic urticaria were improved for long periods after 2 to $3 \frac{1}{2}$ weeks' medication. Four patients with nervous pruritus obtained relief, whereas five other patients required ancillary measures to effect improvement. Among 17 patients with neurodermite, 12 enjoyed suppression of irritation without any apparent local change occurring in the lesion. Five patients with prurigo vulgaris were cured. Itching in lichen ruber planus, psoriasis, and after scabies also responded to antistin.

The dosage Brack used was empirical, and varied from $100-200 \mathrm{mg}$. during the night to $600 \mathrm{mg}$. by mouth through the day. It was also given by intramuscular or slow intravenous injection in doses up to $100 \mathrm{mg}$. A few patients experienced transitory faintness or dizziness, but this was avoided by reducing the dosage or retarding the rate of intravenous injection. One patient suffered tetany-like cramp of hands and fingers on doses exceeding $400 \mathrm{mg}$. daily.

Skouby (1947) treated a group of 43 patients-six with vasomotor rhinitis, 10 with angioneurotic oedema, 15 with urticaria, 12 with asthma. It proved effective in the first three groups only. Thirteen patients suffered from mildly disturbing side-effects, which in seven patients persisted during administration of the drug. Two of the latter preferred to discontinue treatment.

Britton (1947) has used it for 11 patients with urticaria, with some relief in five. Twenty among 54 patients developed unpleasant side-reactions, and in seven they were severe enough to prevent continuation of this mode of treatment. The reactions included nausea (9), drowsiness (4), faintness (3), giddiness (3), headache (2), coughing attacks (2), disorientation (1), depression (1), diarrhoea (1).

\section{Dosage, Mode of Administration, and Control Therapy}

The following results are culled from clinical experience with the drug dating from March, 1947. It has been used, mainly in the out-patient department, on some 87 cases. Administration has of necessity been given in the majority of cases by the oral route, occasionally supplemented by intramuscular injection. Dosage has generally been 
increased until a therapeutic result was achieved, or toxic symptoms supervened, or a dose of $800 \mathrm{mg}$. daily had been given

As a control for the results, the effects of substitution of dummy tablets (suitably flavoured with liq. strychnine, 1/192 gr.) and cessation of therapy were assessed.' In addition, in the non-urticarial pruritic syndromes the effect of a potassium bromide sedacive tonic mixture was compared with that produced by antistin.

Actinic Dermatitis.-Prolonged observation throughout an exceptionally fine summer, with the use of adequate control therapy, did not provide any clear-cut evidence suggesting clinical improvement on antistin in two patients with this disorder, though the drug was pushed to the limits of tolerance. "Benadryl" in maximum tolerated dosage proved equally ineffective.

Cheiropompholyx.-Three patients with cheiropompholyx of constitutional origin obtained no relief from their irritation on antistin $100 \mathrm{mg}$. four times a day by mouth. A similar lack of response followed the use of benadryl.

Chronic Constitutional Eczema.-Compared with adequate control measures, 12 out of 13 patients experienced a moderate amount of relief from their pruritus without any change in the progress of the local manifestations being noted. Its effectiveness did not exceed that of $10 \mathrm{gr}$. $(0.65 \mathrm{~g}$.) of potassium bromide thrice daily in mixture form. Seven patients had previously obtained no assessable benefit on the maximum tolerated dosage of benadryl.

Infantile Eczema.-Eight patients, ranging in age from 4 months to 6 years, unfortunately showed no evidence of relief of irritation, or improvement in the local condition. Dosage ranged from $25 \mathrm{mg}$. four times daily for a 4-monthsold infant to $50 \mathrm{mg}$. four times a day in a 6-year-old child. One child, aged 2 , vomited on two occasions when taking $50 \mathrm{mg}$. thrice daily; there was no recurrence of the vomiting on continuing the same dosage.

Eczematous Conditions with Oedema.-The value of antistin therapy in this group of patients proved more difficult to assess. It would have been preferable to produce a series divided into two comparable groups of patients-one treated by local applications and antistin, and a second by local measures only. Circumstances, and the relatively small number of patients, have not permitted this, but the clinical impression gained was that antistin therapy was a useful adjunct in the treatment of this group. There were nine cases.

Varicose Eczema.-A woman suffered sudden generalization, with marked oedema of face, occlusion of eyes, and very severe pruritus. Antistin was given in $100-\mathrm{mg}$. doses intramuscularly thrice daily for two days, together with the application of lead lotion to the face and zinc cream to the limbs and trunk. The irritation began to diminish in 8 to 12 hours, and the oedema and symptoms had markedly abated in 24 to 36 hours.

Constitutional Eczema.-Three patients who had suffered during a number of years from recurrent attacks of generalized eczema, with oedema of the face and occasionally the arms, were treated in the above manner. The symptoms began to subside in 12 hours, and the oedema in 24 to 36 hours. One patient stated that on previous occasions the attacks, which were treated by similar local measures only, had persisted for longer periods, the oedema generally requiring from 4 to 7 days to abate and the pruritus continuing for a further number of days.

Contact Dermatitis.-Two men, suffering from mercury and cement contact dermatitis, respectively, with swelling of the face, neck, forearms, and hands, associated with confluent vesicular eczema, showed a great reduction in the oedema, diminution of the irritation, and some regression of the eruption after 36 hours. Antistin was given in a 100-mg. dose intramuscularly thrice daily for one day, followed by $200 \mathrm{mg}$. by mouth four times a day. Again, simple local applications were concurrently used.

Two other patients suffering from a similar sensitization dermatitis, but of unknown aetiology, involving the face, responded in an equally satisfactory manner.

Erythema Multiforme.-No beneficial effect was noted in two patients with this syndrome.

Dermatitis Herpetiformis.-A man aged 78 suffering from this disorder did not obtain any relief on an arsenical mixture or $1 / 2$ gr. $(32 \mathrm{mg}$.) of phenobarbitone thrice daily. Antistin-
$100 \mathrm{mg}$. four times a day-induced a mitigation of this symptom, but only during administration of the drug.

Lichen Obtusus Corneus.-A woman who suffered from a patch of lichen obtusus corneus in the left supraclavicular fossa for two years had been treated by occlusion of the area, infiltration with " novocain" and "proctocaine," Grenz rays, and $x$ rays, as well as the usual antipruritic local applications, but without any lasting success. Antistin, $200 \mathrm{mg}$. by mouth, was prescribed for the attacks of irritation. A certain amount of relief followed. Finally the area was infiltrated direct with $100 \mathrm{mg}$. of antistin, no previous local analgesic being given, as antistin itself has a local analgesic action. Some improvement followed, and the manœuvre was repeated in a month's time. A month later the lesion had disappeared completely, and no recurrence has been noted after four months' observation.

Lichen Planus.-Of four patients, two were almost completely relieved of the irritation during ingestion of the tablets. but two derived no benefit. One of these was a man aged 66, who complained of transient dizziness while taking $100 \mathrm{mg}$. four times a day by mouth. The actual course of the disease, and the appearance of the eruption, seemed to be unaffected.

Mycosis Fungoides.-A woman who had suffered from a generalized eczematous condition with marked pruritus for five years, and who ultimately disclosed the true nature of her disorder by developing typical nodules, confirmed histologically, obtained partial relief from her pruritus on this medication without any change in the lesions.

Pemphigus Vulgaris.-Owing to the unsatisfactory state of our knowledge in the treatment of this condition antistin was tried. Though it was administered to the limits of tolerance to two patients no improvement occurred. The effect of benadryl on one of these patients proved equally disappointing.

Senile Pruritus.-Two men, both aged 80, obtained relief. whilst under observation for four and three months respectively, on $100 \mathrm{mg}$. of antistin four times a day by mouth. A woman aged 72 found no reduction in the irritation on a similar dosage. Another man, aged 80 , who obtained no benefit from antistin $100 \mathrm{mg}$. four times daily, suffered a transitory paresis of the left leg and collapsed while taking this dosage.

Pruritus Vulvae et Ani.-It was hoped that antistin would afford some relief in this condition. Among 14 patients suffering from pruritus vulvae or pruritus ani, or both, of idiopathic aetiology, none showed any consistent improvement. Neither did any benefit accompany or follow the use of benadryl in equivalent dosage.

Psoriasis.-A man aged 38 experienced considerable irritation during an exacerbation of his flexural psoriasis. Antistin afforded no relief.

Chronic Urticaria.-Sixteen patients with this disorder were treated over long periods. In 13, urticarial manifestations almost completely disappeared. In the majority this relief was noted 24 to 48 hours after starting treatment. Two more patients suffered mild undisturbing recurrences, and one other on his own assessment showed a $50 \%$ improvement. One derived no benefit. Four of these patients have been free from a recurrence for three to four months following two months' antistin therapy. Four of the persons forming this group also suffered from recurrent attacks of angioneurotic oedema. In one these gradually subsided and disappeared entirely after three months' treatment with antistin; one obtained complete relief when taking the tablets; whilst the other two still had recurrences, but these were milder and less frequent. Nine of these patients had been previously treated with benadryl. Five had developed drowsiness on their respective dosage and preferred to discontinue this treatment and retain their urticaria. Three patients who did not respond had only been given a maximal dose of $100 \mathrm{mg}$. thrice daily. One patient was equally well controlled on benadryl and " anthisan."

Urticaria Papulosa.-Six children suffering from this condition did not experience any mitigation of their itch on doses up to $100 \mathrm{mg}$. four times a day. Two children showed a reduction in the tendency to scratch. One child, aged 3 , developed transient convulsions on $100 \mathrm{mg}$. three times a day. Cessation of therapy was accompanied by complete resolution of this disturbing symptom. 


\section{Surveillance and Investigations}

Twenty-three patients had serial blood counts performed during two to three months' therapy. No significant variations were noted. Seventeen patients had serial blood-pressure estimations performed in the out-patient department over a similar period; six of these were over 70 years old, with arteriosclerosis and mild systolic and diastolic hypertension. Antistin therapy did not appear to cause any permanent significant alteration in the readings. Repeated urinalysis in 30 patients without albuminuria or glycosuria initially did not reveal the development of either during the course of treatment.

\section{Discussion}

Antistin is one of the three antihistamine substances available in Great Britain at the moment, the other two being benadryl and anthisan. The indications for the use of the first two in dermatology are, in my experience, identical, and there is little to choose between them in efficacy. One feature concerning the effects of benadryl therapy has repeatedly been observed-notably its high percentage of toxic manifestations which, though mainly mild, are sufficient to limit its sphere of usefulness. Among 120 patients Stroh (1946) found that $14.2 \%$ suffered from undesirable side-effects. Slater and Francis (1946) recount an incident in which the driver of an electric truck lost control over his machine while taking benadryl. Nervousness, incoordination, and epileptiform convulsions occurred in a child aged $3 \frac{1}{2}$ years who was being treated for hayfever (Weil, 1947). Of McElin and Horton's (1945) patients $60 \%$ complained of sleepiness, $16 \%$ of dizziness, and $14 \%$ of nervousness. Koelsche, Prickman, and Carryer (1945) state that 25 among 83 patients complained of drowsiness. O'Leary and Farber $(1945,1946)$ noted that $33 \%$ of their patients suffered from toxic reactions. Friedlander (1946) found a similar incidence of side-effects. Again, among 696 patients treated with benadryl, 366 instances of drowsiness occurred (Ann. Allergy, 1946).

In view of its somnolent and depressing effect I am reluctant to order benadryl for the motor driver, the skilled mechanic and operator, or others daily performing potentially dangerous tasks requiring a high degree of mental alertness and co-ordination. In my experience antistin has given equally good therapeutic results with a much lower incidence of untoward reactions.

There is, however, only a limited field of usefulness for this type of drug in dermatology ; they effect a symptomatic alleviation, but not a cure. Those marketed to date may all cause immediate unpleasant side-reactions, and their remote toxicity has yet to be fully assessed. In some instances the amount of relief afforded justifies their use ; in other disorders the same, or greater, relief can be obtained by well-tried, innocuous, and cheaper methods. In my experience, indications for the use of antistin are as follows :

1. Urticaria and Angioneurotic Oedema.-It affords marked symptomatic relief in the treatment of this condition while specific or non-specific curative measures are being used. It is often useful in reducing the polysusceptibility of certain skins when testing by scratch or intradermal methods.

2. Eczematous Conditions with Oedema.-It offers a useful addition to the dermatologist's armarium for expediting recovery.

3. Itching in Other Skin Conditions.-Occasionally it may prove helpful for mitigation of itching when the usual methods prove ineffective.

\section{Undesirable Reactions}

The following table reveals the incidence of unwanted manifestations found among 87 patients.

$$
\text { Sympioms }
$$

Dizziness and lightheadedress
No patient was prevented from obtaining benefit from this medication by the occurrence of minor untoward symptoms. If improvement occurred together with toxic manifestations a reduction in dosage relieved the unwanted symptoms without affecting the desired result.

The child who developed convulsions on $100 \mathrm{mg}$. three times a day by mouth also had threadworms, but on cessation of antistin therapy without treatment of her worms she suffered no recurrence. I am unable to state whether the heavy dosage of antistin was the sole cause of the convulsions or an additional factor to the worms in producing convulsions, or whether the child was a potential epileptic who was stimulated to the convulsing threshold by this medication. After this incident I did not give more than $200 \mathrm{mg}$. daily to a child of this age.

The only instance of vomiting noted was in a child aged 2 years receiving $150 \mathrm{mg}$. by mouth daily. It occurred on two occasions after taking half a tablet, but during the course of subsequent unreduced therapy there was no recurrence.

The patient who collapsed was a man aged 80 with arteriosclerosis in his brachial, radial, and retinal vessels, and a blood pressure of $170 / 95$. He appeared amazingly fit, both mentally and physically, but whilst out walking suddenly fell in the street, with a sensation of having lost the use of his left leg. There were no other associated symptoms. He was aided home and recovered completely within half an hour. I saw him two weeks after the incident, and there was no evidence of any impairment of his nervous system. His pruritus had been unrelieved.

Nocturnal disorientation occurred in a man aged 76 on three occasions about an hour after taking his evening dose of $100 \mathrm{mg}$. of antistin by mouth. He was suffering from generalized eczema and had arteriosclerosis with a fairly constant blood pressure around 140/85. On one occasion he was found wandering aimlessly in a dazed condition round the ward.

It is to be noted that the major ill effects occurred either in extreme youth or in extreme age.

\section{Summary}

The effect of antistin on a number of skin diseases has been noted. It afforded symptomatic relief in urticaria and angioneurotic oedema, in some eczematous eruptions with oedema, and occasionally in other itching dermatoses. Sometimes it produced the desired result when benadryl failed. Undesirable side effects occurred in 14 out of 87 patients-a relatively low incidence for this type of drug at the present time. The only serious undesirable secondary symptoms occurred either in early childhood or in the very old.

I wish to thank my numerous colleagues who kindly referred their patients to me, and Mr. A. R. G. Chamings, of Ciba, Ltd., who provided the drug and much useful information.

\section{REFERENCES}

Annals of Allergy, 1946, 4, 466.

Brack, W. (1946). Schweiz. med. Wschr., 76, 316.

Britton, C. J. C. (1947). Lancet, 2, 870.

Friedlander, A. S. (1946). Amer. J. med. Sci., 212, 185.

Koelsche, G. A., Prickman, L. E., and Carryer, H. M. (1945). Proc. Mayo Clin., 20, 432.

McElin, T. W., and Horton, B. T. (1945). Ibid., 20, 417

Meier, R., and Bucher, K. (1946a). Experientia, 2, 140.

Meier, R., (1946b). Schweiz. med. Wschr., 76, 294.

O'Leary, P. A., and Farber, E. M. (1945). Proc. Mayo Clin., $20,429$. (1946). Ibid., 21, 295.

Schindler, O. (1946). Schweiz. med. Wschr., 76, 300

Skouby, A. P. (1947). Nord. Med., 34, 1022.

Slater, B. J., and Francis, N. (1946). J. Amer. med. Ass., 132, 212.

Staub, H. (1946). Helv. physiol. pharmacol. Acta, 4, 539.

Stroh, J. E. (1946). Northw. Med., Seattle, 45, 846.

Weil, H. R. (1947). J. Amer. med. Ass., 133, 393. 